\title{
The Role of Viscosity in TATB Hot Spot Ignition
}

L. E. Fried, L. Zepeda-Ruis, W. M. Howard, F. Najjar, J. E. Reaugh

August 3, 2011

17th APS Shock Compression of Condensed Matter Conference

Chicago, IL, United States

June 26, 2011 through July 1, 2011 
This document was prepared as an account of work sponsored by an agency of the United States government. Neither the United States government nor Lawrence Livermore National Security, LLC, nor any of their employees makes any warranty, expressed or implied, or assumes any legal liability or responsibility for the accuracy, completeness, or usefulness of any information, apparatus, product, or process disclosed, or represents that its use would not infringe privately owned rights. Reference herein to any specific commercial product, process, or service by trade name, trademark, manufacturer, or otherwise does not necessarily constitute or imply its endorsement, recommendation, or favoring by the United States government or Lawrence Livermore National Security, LLC. The views and opinions of authors expressed herein do not necessarily state or reflect those of the United States government or Lawrence Livermore National Security, LLC, and shall not be used for advertising or product endorsement purposes. 


\title{
THE ROLE OF VISCOSITY IN TATB HOT SPOT IGNITION Laurence E. Fried $^{1}$, Luis Zepeda-Ruis ${ }^{1}$, W. Michael Howard ${ }^{1}$, Fady Najjar, John E. Reaugh ${ }^{1}$ \\ ${ }^{I}$ Physical and Life Sciences Directorate, Weapons and Complex Integration Directorate ${ }^{2}$, Lawrence Livermore National Laboratory, 7000 East Avenue, Livermore CA 94551
}

\begin{abstract}
The role of dissipative effects, such as viscosity, in the ignition of high explosive pores is investigated using a coupled chemical, thermal, and hydrodynamic model. Chemical reactions are tracked with the Cheetah thermochemical code coupled to the ALE3D hydrodynamic code. We perform molecular dynamics simulations to determine the viscosity of liquid TATB. We also analyze shock wave experiments to obtain an estimate for the shock viscosity of TATB. Using the lower bound liquid-like viscosities, we find that the pore collapse is hydrodynamic in nature. Using the upper bound viscosity from shock wave experiments, we find that the pore collapse is closest to the viscous limit.
\end{abstract}

Keywords: Hydrodynamics, reactive flow, shocked TATB, pore collapse, Cheetah, ALE3D, viscosity. PACS: 44.35.+c, 62.50.Ef, 95.30.Lz.

\section{INTRODUCTION}

Solid plastic-bonded high-explosive (HE) materials consist of crystals that are usually between 10 and 100 microns, with micron-sized impurities and pores embedded inside the crystals. These voids increase the ease of shock initiation by generating high-temperature regions during their collapse that might lead to ignition. Understanding the mechanisms of hot-spot initiation in $\mathrm{HE}$ materials has had significant research interest in the literature due to safety, reliability and development of new insensitive munitions. Bowden \& Yoffe [1] systematically studied the role of void collapse. The main mechanisms of hot spot formation identified consist of adiabatic compression of small entrapped bubbles of gas, friction due to confining surfaces or extraneous grit particles, and intercrystalline friction of the explosive particles, and viscous heating of rapidly flowing explosive material. Frey [2] reviewed the theoretical effects concerning the role of voids in explosive initiation and ignition. Mader [3] studied numerically the shock initiation of detonation in nitromethane, liquid TNT and single-crystal PETN.
In the present paper, we study the role of shock viscosity in determining pore collapse dynamics in the insensitive high explosive TATB. For simplicity, we consider spherical pores in the current study. Shock viscosity is a simple way of treating plastic dissipation in materials under shock loading. In the absence of detailed data on the plastic response of TATB under compressive loading, we develop upper and lower bounds on the possible shock viscosities of TATB, and then calculate the pore collapse under the bounding conditions.

The pore collapse dynamics are expected to be controlled by the dimensionless Reynolds number [4]

$$
R=\frac{d \sqrt{\rho_{s} P_{s}}}{8 \eta}
$$

Here, $d$ is the pore diameter, $\rho_{\mathrm{s}}$ is the density of the shocked TATB, $P_{s}$ is the shock pressure, and $\eta$ is the shock viscosity. When $\mathrm{R}$ is much greater than 1 , the pore collapse is expected to be jet-like (hydrodynamic) in nature. In this case, a jet is formed on the axis of symmetry of the pore. Ignition is started by shock heating when the jet 
impacts the opposite wall of the pore. On the other hand, when $\mathrm{R}$ is much less than 1 , the pore collapse is expected to be viscous in nature. In this limit the pore retains a roughly spherical shape as it collapses. Chemistry is expected to occur due to viscous heating during the collapse.

The reliable experimental determination of shock viscosity is difficult. The shock viscosity can be determined from the relation [5]

$\eta=S P_{s} \Delta t / 4$

where $\mathrm{S}$ is the slope of the Us-Up shock Hugoniot, and $\Delta \mathrm{t}$ is the rise time of the shock. The "true" shock viscosity at the grain scale is determined by the rise time of the shock in a single crystal. The rise time of the shock in a composite should be greater than that in a single crystal due to multiple interacting nano-shocks in the composite. Measured shock rise times can be in the picosecond regime, making the determination of shock viscosity experimentally challenging.

Hambir et al. [4] studied pore collapse in PMMA under shock pressures of $\sim 4 \mathrm{GPa}$. They inferred a shock viscosity of $3 \mathrm{~Pa}-\mathrm{s}$ and a Reynolds number of 0.1 for $100 \mathrm{~nm}$ voids. This supports the viscous pore collapse model. Some prior studies [6] have proposed significantly higher shock viscosities for high explosives, in the neighborhood of $100 \mathrm{~Pa}-\mathrm{s}$.

There are some reasons to believe that pore collapse in reacting insensitive high explosives may have a jet-like mechanism. Reacting hot spots in insensitive high explosives are larger in diameter than those in conventional high explosives for the same shock strength. Secondly, the pressure thresholds for reaction in insensitive high explosives are several times higher than those in conventional (e.g. HMX-based) high explosives. This argues that hydrodynamic pore collapse may be important in IHE.

We have estimated the shock viscosity of TATB using gauge data for detonating PBX 9502[7]. PBX 9502 is $95 \%$ TATB by weight, with $5 \%$ of a binder material added. Sheffield et al. [7] found a shock rise time of less than $0.3 \mathrm{~ns}$. They also found a von Neumann spike pressure of 37 $\mathrm{GPa}$ and a value of 1.7 for $\mathrm{S}$. Using relation (2), we find that $\eta$ is roughly $4.5 \mathrm{~Pa}-\mathrm{s}$. We regard this as an upper limit on the shock viscosity of TATB, since the experiment was performed on a multicrystalline composite.

\section{ATOMISTIC SIMULATIONS}

In order to evaluate a lower bound on the shock viscosity, we have performed molecular dynamics simulations on liquid TATB. Under this hypothesis, TATB melts upon shock loading. We note that liquid TATB is not chemically stable, which prevents experimental study. TATB is known to melt with decomposition at a temperature of $623 \mathrm{~K}$. Cheetah calculations indicate a temperature of $1100 \mathrm{~K}$ for TATB at its von Neumann spike condition. Therefore, it is at least plausible that TATB melts upon shock loading prior to reaction.

In the molecular dynamics simulations, the force field of Gee et al. [8] was used. A nonequilibrium molecular dynamics approach was employed, where the simulation cell underwent shear deformation at a fixed strain rate. The shear viscosity was determined from the magnitude of the shear stress induced by the strain. Simulations were conducted as a function of temperature and of shear rate. In Fig. 1, we show the viscosity of TATB as a function of temperature at a fixed density of $1.65 \mathrm{~g} / \mathrm{cc}$. This density was chosen as an estimate of the density of the zero pressure liquid phase.

As expected, the shear viscosity decreases with increasing temperature. The shear viscosity as a function of shear rate is shown in Fig. 2. Our simulations indicate that the viscosity of liquid TATB is very sensitive to the strain rate. We expect that a micron-sized void should collapse on a time scale of about $1 \mathrm{~ns}$. Therefore, a relevant shear rate for void collapse is about $10^{9}$. We have selected a value of $0.025 \mathrm{~Pa}-\mathrm{s}$ as a representative viscosity for liquid TATB under shock loading. Future study will investigate the pressure dependence of the shear viscosity in the liquid phase. 


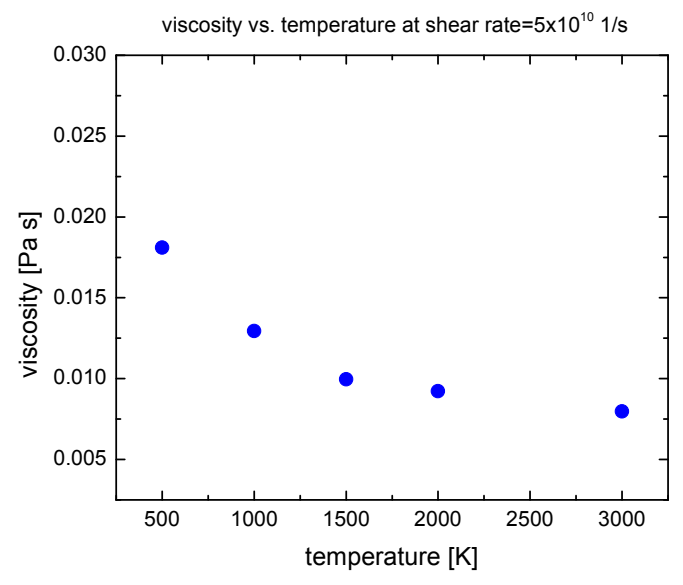

Figure 1. The calculated shear viscosity of liquid TATB as a function of temperature at $1.65 \mathrm{~g} / \mathrm{cc}$ and a strain rate of $5 \times 10^{10} \mathrm{~s}^{-1}$.

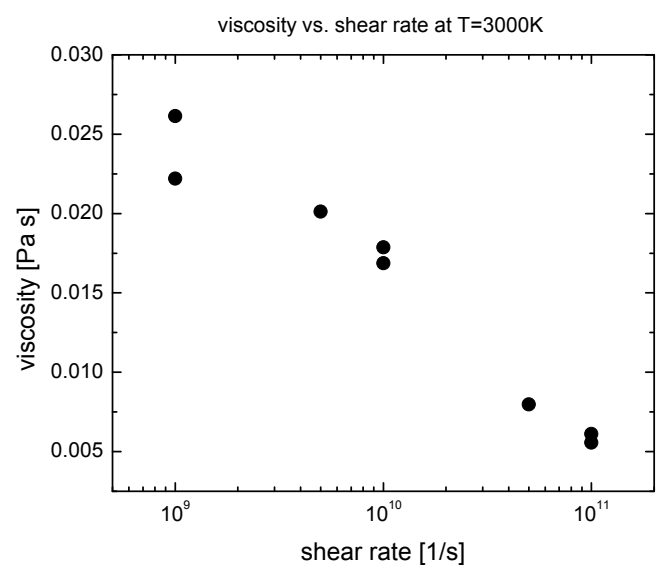

Figure 2. The calculated shear viscosity of liquid TATB as a function of shear strain rate at a temperature of $3000 \mathrm{~K}$ and a density of $1.65 \mathrm{~g} / \mathrm{cc}$.

\section{CONTINUUM SIMULATIONS}

The multiphysics software, ALE3D [9], is used to perform these simulations and consists of several physics modules, including hydrodynamics and thermal transport. The mathematical formulation is based on an Operator-Split method and invokes an arbitrary Lagrangian-Eulerian (ALE) approach in
3-D/2-D configuration. The thermo-chemistry solver, Cheetah [10], is tightly coupled to ALE3D and properly models appropriate kinetic reaction mechanisms, obtained from MD computations.

The current configuration is represented by a continuum material of an insensitive HE material, TATB, where an embedded defect consisting of a spherical air-filled pore is present with an initial diameter, $\mathrm{d}_{\mathrm{p}}$. The cylindrical computational domain consists of a rectangular grid with length and radius, $\mathrm{L}=16 \mu \mathrm{m}$, and $\mathrm{R}=8 \mu \mathrm{m}$, respectively. The pore diameter $\mathrm{d}$ was $4 \mu \mathrm{m}$. The following boundary conditions are imposed: a solid wall on the left boundary, and non-reflective outflow condition on the right boundary, with symmetric conditions on the top and bottom boundaries. Reverse ballistics are imposed initially where a uniform $\mathrm{x}$-velocity is set. We chose an initial velocity of $2.4 \mathrm{~mm} / \mu \mathrm{s}$, which led to a shock pressure of $33 \mathrm{GPa}$ prior to pore collapse. Atmospheric conditions are chosen for the pressure field and temperature ( $\mathrm{p}=1 \mathrm{~atm}$ and $\mathrm{T}=298 \mathrm{~K})$.

The chemical reactions for TATB are obtained from MD simulations where a 4-step approach was identified, making TATB into products; while the constitutive relation for air follows a Quotidian EOS (QEOS) [11]. Hence, the current hydrosimulations consist of a three-way coupled formalism, including the hydrodynamics and thermal transport modules in ALE3D, and chemical reactions using Cheetah [10].

We have performed two simulations: one with the upper viscosity bound of $4.5 \mathrm{~Pa}-\mathrm{s}$, and one with the lower viscosity bound of $0.025 \mathrm{~Pa}-\mathrm{s}$. Results are shown in Fig. 3. In the simulation with the upper viscosity bound, we find that ignition occurs during compression, similar to the viscous collapse limit. The collapse is asymmetric, however, indicating that hydrodynamic effects are still present. In the simulation with the lower viscosity bound, however, we see features indicative of the hydrodynamic pore collapse model. Ignition is observed to occur upon jet impact. We conclude that hydrodynamic features could be important in the pore collapse of TATB-containing explosives. 

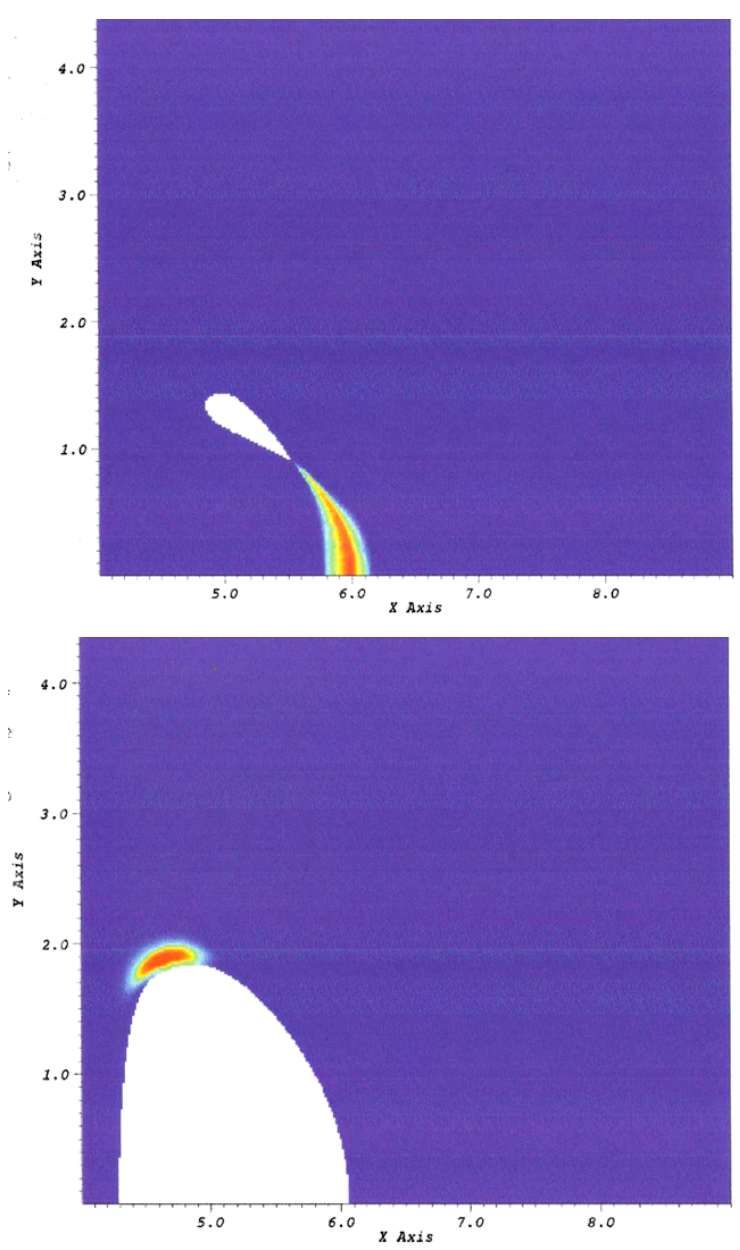

Figure 3. The mass fraction of TATB final decomposition product is shown immediately after pore collapse for the simulation with liquid-like shear viscosity. Red indicates that all the TATB has been converted to initial product. Blue indicates no initial product. Distance is shown in microns.

\section{ACKNOWLEDGEMENTS}

This work was performed under the auspices of the U.S. Department of Energy by Lawrence Livermore National Security under contract No. DE-AC52-07NA27344. The authors acknowledge the numerous discussions with C. Tarver, A. L. Nichols III, and N. Barton.

\section{REFERENCES}

1. Bowden, F.P., \& Yoffe, A.D., Initiation and Growth of Explosions in Liquids and Solids, Cambridge Univ. Press, New York, 1951.

2. Frey, R.B., "Cavity Collapse in Energetic Materials," Proceedings-Eighth Symposium on Detonation, 385-393, 1985.

3. Mader, C., "Shock and Hot Spot Initiation of Homogeneous Explosives," Phys. Fluids, 6(3): 375381, 1963.

4. Hambir, S. A., Kim, H., Dlott, D. D., "Real time ultrafast spectroscopy of shock front pore collapse", J. App. Phys., 90, 5139(2001).

5. Grady, D. E., "Shock-wave compression of brittle solids" Mech. Mater., 46, 181-203(1998).

6. Menikoff, R. and Sewell, T. D., "Constituent properties of HMX needed for mesoscale simulation", Combust. Theory and Modeling, 6, 103-125(2002).

7. Sheffield, S. A., Bloomquist, D. D., and Tarver, C. M., "Subnanosecond measurments of detonation fronts in solid high explosives", J. Chem. Phys., 80, 3831(1984).

8. Gee, R. H., Roszak, S., Balasubramanian, K., and Fried, L. E., "Ab initio based force field and molecular dynamics simulations of crystalline TATB”, J. Chem. Phys., 120, 7059-7066 (2004).

9. Nichols, A.L. et al., "ALE-3D User's Manual”, LLNL Technical Report, UCRL-MA-152204, 2007.

10. Fried, L.E., \& Howard, M.W., "An Accurate Equation of State for the Exponential-6 Fluid Applied to Dense Supercritical Nitrogen," J. Chem. Physics, 109(10):7338-7349, 1998.

11. More, R.M., et al., "New Quotidian Equation of State (QEOS) for Hot Dense Matter", Phys Fluids, 31(10):3059-3078, 1988. 